\title{
Renormalization And Lorentz Symmetry Violation
}

\author{
Damiano Anselmi* \\ Dipartimento di Fisica "E. Fermi", Università di Pisa, \\ Largo B. Pontecorvo 3, I-56127 Pisa, Italy, \\ and INFN, Sezione di Pisa, Italy \\ E-mail: damiano.anselmiedf.unipi.it
}

\begin{abstract}
If Lorentz symmetry is violated at high energies, interactions that are usually non-renormalizable can become renormalizable by weighted power counting. We present a CPT invariant, Lorentz violating extension of the Standard Model containing two scalar-two fermion interactions (which can explain neutrino masses) and four fermion interactions (which can explain proton decay). The presence of four fermion vertices allows us to argue that the known low energy physics can be reproduced also if the elementary scalars are suppressed, in the Nambu-Jona-Lasinio spirit. The scalarless model predicts relations among otherwise independent parameters of the Standard Model.
\end{abstract}

Workshop on Continuum and Lattice Approaches to Quantum Gravity September 17-19 2008

Brighton, University of Sussex, United Kingdom

\footnotetext{
* Speaker.
} 


\section{Introduction}

Lorentz symmetry is a basic ingredient of the Standard Model of particle physics. Nevertheless, several authors, inspired by different considerations, have suggested that Lorentz symmetry and CPT could be broken at very high energies or very large distances. The problem of Lorentz violation has attracted a lot of interest, in cosmology, astrophysics, high-energy physics. If Lorentz symmetry were not exact our understanding of Nature would change considerably. On the other hand, the parameters of the Lorentz violating Standard-Model extension [1] have been tested with great precision [2] and found in very good agreement with Lorentz invariance in a quite wide range of energies.

Massive gravity plays an important role in the study of large-distance modifications of the gravitational force [3]. Certain problems, such as the vDVZ discontinuity, appear less sever if the graviton mass term violates Lorentz symmetry [4]. The possibility of high-energy Lorentz violation is also open $[5,1]$ and has inspired several investigations about the new physics that could emerge [6].

Lorentz violating quantum field theory can be useful for several purposes. It contains nonrelativistic field theory, and has applications to nuclear physics [7], effective field theory [8, 9], critical phenomena [10], cosmology [11] and possibly high energy physics. In condensed matter physics it can describe higher temperature superconductors, ferroelectric liquid crystals, polymers and magnetic materials $[12,13]$.

In this talk we focus on the relation between Lorentz symmetry, or Lorentz violation, and renormalizability. The set of ordinary power-counting renormalizable theories is considerably restricted by the assumptions of unitarity, locality, causality, stability, polynomiality and Lorentz invariance. If we relax one or some of these assumptions we can certainly enlarge the set of renormalizable theories. However, usually the enlargement is too wide. For example, there exist an infinite set of renormalizable non-unitary theories. Improving the behavior of propagators at large momenta with higher-derivative kinetic terms [14] it is possible to define a renormalizable higherderivative version of every theory, including gravity [15]. Relaxing locality can in principle make every theory finite, smoothing away the small distance singularities that originate the UV divergences [16]. Relaxing Lorentz invariance, instead, appears to be more promising. Since we want to change the high-energy behavior of the theory in an essential way, the Lorentz violation cannot be spontaneous, but must be explicit.

The UV behavior of propagators can be improved with the help of higher spatial derivatives. Higher-time derivatives are not allowed, by unitarity. If the vertices and quadratic terms are arranged according to a certain "weighted power counting" criterion, which weighs time and space differently, no higher time derivatives are generated by renormalization. The set of consistent theories is still very restricted, yet considerably larger than the set of Lorentz invariant theories. Interactions that are not renormalizable by ordinary power counting, such as two scalar-two fermion vertices and four fermion vertices, can become renormalizable by weighted power counting. All other properties, namely unitarity, locality, causality, stability and polynomiality, can be preserved. Consistent renormalizable models exist in arbitrary spacetime dimensions. In non-gauge theories several types of Lorentz breakings are allowed $[17,18]$. On the other hand, the presence of gauge interactions puts a severe restriction, because it selects a unique type of Lorentz breaking, the one 
that splits spacetime into space and time.

The quadratic terms that contain higher space derivatives, as well as the vertices of higher dimensions, are multiplied by inverse powers of a scale $\Lambda_{L}$. Despite the presence of the dimensionful parameter $\Lambda_{L}$ certain models, the strictly-renormalizable ones in the weighted sense, have a weighted scale invariance, which is anomalous at the quantum level. Once Lorentz symmetry is violated at high energies, its low-energy recovery is not automatic, because renormalization makes the low-energy parameters run independently. To recover it, we have to advocate a fine tuning that relates those parameters in a suitable way. It is not apparent how to justify this fine-tuning, unless the Lorentz invariant surface is RG stable [19].

Lorentz invariance can be violated preserving or not preserving CPT. We concentrate the major part of our attention on the "minimal breaking" of Lorentz symmetry, which preserves CPT and invariance under space rotations. It is interesting to inquire what physics beyond the Standard Model emerges in the approach described so far, assuming that Lorentz symmetry is violated by terms of higher dimensions and restored at low energies. In particular, we can formulate [20] a Standard Model extension with the following properties: it is CPT invariant, but Lorentz violating at high energies, it is unitary and renormalizable by weighted power counting; it contains the vertex $(L H)^{2} / \Lambda_{L}$ [21], which gives Majorana masses to the neutrinos after symmetry breaking, but no right-handed neutrinos, nor other extra fields; it contains four fermion vertices, which can explain proton decay. If neutrino masses are due to the Lorentz violation, then the scale $\Lambda_{L}$ of Lorentz violation is about $\sim 10^{14} \mathrm{GeV}$. Below that scale, Lorentz symmetry is recovered.

The Standard-Extended Model has two "weighted" dimensions, which means that at high energies its power counting resembles the one of a two-dimensional quantum field theory. In particular, only the four fermion vertices are strictly renormalizable, while the gauge and Higgs interactions are super-renormalizable. This means that at energies $\gg \Lambda_{L}$ all gauge bosons and the Higgs field become free and decouple, and what remains is a (Lorentz violating) four fermion model in two weighted dimensions. It is then natural to inquire what physical effects are induced, at lower energies, by a dynamical symmetry breaking mechanism, in the Nambu-Jona-Lasinio spirit [22]. If we suppress the elementary scalar field in the model of [20], we obtain a simpler model that is candidate to reproduce the observed low energy physics, predict relations among otherwise independent parameters, and possibly predict new physics detectable at LHC.

In flat space, we do not find inconsistencies with the basic principles of quantum field theory. Causality is obeyed à la Bogoliubov [18], which does not require light cones, but only past and future. At large distances, where Lorentz symmetry is restored, spacelike separated operators do commute, but at small distances they can have non-trivial commutators. The Källen-Lehmann spectral decomposition is fine [18]. The Goldstone theorem can also be proved [23]. Anomalies are independent of the Lorentz violation and the Adler-Bardeen theorem can be derived [20], ensuring, as usual, anomaly cancellation to all orders in a suitable subtraction scheme.

Despite its internal consistency, two aspects of Lorentz violating quantum field theory might be viewed as weaknesses. We have already mentioned that a fine-tuning is necessary to recover Lorentz symmetry at low energies. The deviations from the Lorentz symmetric theory are parametrized by $\Lambda_{L}$ and a number of dimensionless quantities $\delta_{L}$. Agreement with experiments requires that several $\delta_{L}$ 's have very small values. In particular, the photon and electron dispersion relations are modified, and non-trivial refractive indices are predicted at energies $\gtrsim \Lambda_{L}$, if the $\delta_{L}$ 's are of 
order one. Several experiments have put bounds on the scale of Lorentz violation. Some authors [24] claim that the violation can be pushed beyond the Planck scale. However, agreement with experiments can also be achieved with a lower $\Lambda_{L}$, if suitable $\delta_{L}$ 's are not of order 1. Our StandardExtended Model contains sufficiently many free parameters to accommodate present observations. If $\Lambda_{L}$ is around the Planck mass, most $\delta_{L}$ 's can be of order 1. If $\Lambda_{L}$ is much lower, say around $10^{14} \mathrm{GeV}$, then some $\delta_{L}$ 's have to be as small as $10^{-10}$. Yet, the model is predictive in the sense that it contains a finite number of free parameters, so at some stage it will be possible to confirm it or prove it wrong.

The other potential weakness is that our model is not unique. There exist infinitely many, more involved, versions. No upper bound on the number of higher space derivatives seem to follow from first principles. On the contrary, Lorentz invariant quantum field theory is much more restrictive, because there the upper bound on the number of time derivatives, due to unitarity, implies an identical upper bound on the number of space derivatives. This means that some aspects of Lorentz violating quantum field theory are still mysterious. Yet, even this second aspect of Lorentz violating quantum field theory does not ensure that Lorentz symmetry is exact at arbitrarily high energies, because it does not affect internal consistency.

We have worked exclusively in flat space. The ultimate fate of Lorentz violating quantum field theory depends very much on gravity. When gravity is turned on the problem becomes more subtle, since the symmetry that must be broken is local, not global. And it must be broken explicitly, not spontaneously. A naive breaking of Lorentz invariance breaks also diffeomorphisms, therefore unitarity. This is the approach of [25], which we do not think is sound (see for example [26]). Without entering into details, we just say that there must exist a better way to pursue the quantization of gravity other than breaking unitarity.

There also have been interesting attempts to derive gravity from a spontaneous breaking of local Lorentz symmetry [27].

In the first part, where we discuss the technical aspects of renormalization, we mainly work in Euclidean space. When we study the Standard-Extended Model we turn to Minkowski space.

\section{Weighted power counting}

For definiteness, we consider models where the $d$-dimensional spacetime manifold $M_{d}$ is split into the product $M_{\widehat{d}} \times M_{\bar{d}}$ of two submanifolds, a $\widehat{d}$-dimensional submanifold $M_{\widehat{d}}$, containing time and possibly some space coordinates, and a $\bar{d}$-dimensional space submanifold $M_{\bar{d}}$. The $d$-dimensional Lorentz group $S O(1, d-1)$ is broken to a residual Lorentz symmetry $S O(1, \widehat{d}-1) \times$ $S O(\bar{d})$. The generalization of our arguments to the most general breaking is straightforward [17].

Each momentum $p$ is split into "first" components $\widehat{p}$, which live in $M_{\widehat{d}}$, and "second" components $\bar{p}$, which live in $M_{\bar{d}}: p=(\widehat{p}, \bar{p})$. The spacetime index $\mu$ is split into hatted and barred indices as well: $\mu=(\widehat{\mu}, \bar{\mu})$. Notations such as $\widehat{p}_{\widehat{\mu}}, \widehat{p}_{\mu}$ and $p_{\widehat{\mu}}$ refer to the same object, as well as $\bar{p}_{\bar{\mu}}, \bar{p}_{\mu}$, $p_{\bar{\mu}}$.

We say that $P_{k, n}(\widehat{p}, \bar{p})$ is a weighted polynomial in $\widehat{p}$ and $\bar{p}$, of degree $k$ and weight $1 / n$, where $k$ is a multiple of $1 / n$, if $P_{k, n}\left(\xi^{n} \widehat{p}, \xi \bar{p}\right)$ is a polynomial of degree $k n$ in $\xi$. Clearly,

$$
P_{k_{1}, n}(\widehat{p}, \bar{p}) P_{k_{2}, n}(\widehat{p}, \bar{p})=P_{k_{1}+k_{2}, n}(\widehat{p}, \bar{p}) .
$$


We say that $H_{k, n}(\widehat{p}, \bar{p})$ is a homogeneous weighted polynomial in $\widehat{p}$ and $\bar{p}$, of degree $k$ and weight $1 / n$, if $H_{k, n}\left(\lambda \widehat{p}, \lambda^{1 / n} \bar{p}\right)=\lambda^{k} H_{k, n}(\widehat{p}, \bar{p})$. It is immediate to prove that a weighted polynomial $P_{k, n}$ of degree $k$ can be expressed as a linear combination of homogeneous weighted polynomials $H_{k^{\prime}, n}$ of degrees $k^{\prime} \leq k$.

Consider a generic scalar field theory with a propagator defined by the quadratic terms

$$
\mathscr{L}_{\text {free }}=\frac{1}{2}(\widehat{\partial} \varphi)^{2}+\frac{1}{2} \sum_{j=0}^{n} \frac{a_{j}}{\Lambda_{L}^{2 j-2}}\left(\bar{\partial}^{j} \varphi\right)^{2}
$$

(in Euclidean space). Up to total derivatives it is not necessary to specify how the $2 j$ derivatives $\bar{\partial}$ contract among themselves. The number $n$ plays a crucial role, since $2 n$ is the highest power of $\bar{\partial}$ that appears in the quadratic terms of the lagrangian. The coefficient of the term $\left(\bar{\partial}^{n} \varphi\right)^{2}$ must be positive, to have an action bounded from below in the Euclidean framework or, equivalently, an energy bounded from below in the Minkowskian framework. We normalize $a_{n}$ to 1 . The propagator reads

$$
\frac{1}{\widehat{k}^{2}+\sum_{j=0}^{n-1} a_{j} \frac{\left(\bar{k}^{2}\right)^{j}}{\Lambda_{L}^{2 j-2}}+\frac{\left(\bar{k}^{2}\right)^{n}}{\Lambda_{L}^{2 n-2}}} .
$$

To study renormalization, the quadratic terms proportional to $a_{j}, j<n$, can be considered as "interactions" (two-leg vertices) and treated perturbatively. Indeed, the counterterms depend polynomially on such parameters. This property generalizes the well-known polynomiality of counterterms in the masses, and can be proved observing that the integral associated with a graph becomes overall convergent when it is differentiated a sufficient number of times with respect to the $a_{j}$ 's with $j<n$.

Thus we can assume that the propagator is

$$
\frac{1}{\widehat{k}^{2}+\frac{\left(\bar{k}^{2}\right)^{n}}{\Lambda_{L}^{2 n-2}}}
$$

and treat every other terms as vertices. Green functions calculated with (2.2) are IR-smooth, provided we choose non-exceptional external momenta. Thus, the propagator we use is the inverse of a weighted homogeneous polynomial of degree 2 and weight $1 / n$.

Perturbative unitarity demands that no higher-time derivative be present, both in the kinetic part and in the vertices. We need to prove that no higher time derivatives are turned on by renormalization.

We label the vertices that have $N \varphi$-legs with indices $\alpha$, in such a way that each vertex defines a weighted homogeneous monomial in the momenta of the fields. We call

$$
\delta_{N}^{\alpha}=p_{1}+\frac{p_{2}}{n}
$$

the weighted degree of the $\alpha$ th vertex with $N \varphi$-legs and symbolically write such vertex as $\left[\widehat{\partial}^{p_{1}} \bar{\partial}^{p_{2}} \varphi^{N}\right]_{\alpha}$. The lagrangian can be written as

$$
\mathscr{L}_{(\widehat{d}, \bar{d})}=\frac{1}{2}(\widehat{\partial} \varphi)^{2}+\frac{1}{2 \Lambda_{L}^{2 n-2}}\left(\bar{\partial}^{n} \varphi\right)^{2}+\sum_{N, \alpha} \frac{\lambda_{N, \alpha} g^{N-2}}{N ! \Lambda_{L}^{N(d / 2-1)+p_{1}+p_{2}-d}}\left[\widehat{\partial}^{p_{1}} \bar{\partial}^{p_{2}} \varphi^{N}\right]_{\alpha} .
$$


The couplings $\lambda_{N, \alpha}$ are dimensionless and weightless. We have attached an extra dimensionless, but possibly weightful constant $g$ to each $\varphi$-leg, save two of them. We define $g$ as the constant with maximum weight that can fit into the structure (2.3). It will be useful to study super-renormalizable theories.

Consider a Feynman graph $G$ made of $L$ loops, $E$ external legs, $I$ internal legs and $v_{N}^{\alpha}$ vertices of type $N, \alpha$. The integral associated with $G$ has the form

$$
\mathscr{I}_{G}(k)=\int \frac{\mathrm{d}^{L \widehat{d}} \widehat{p}}{(2 \pi)^{L \hat{d}}} \int \frac{\mathrm{d}^{L \bar{d}} \bar{p}}{(2 \pi)^{L \bar{d}}} \prod_{i=1}^{I} \mathscr{P}_{-2, n}^{(i)}(p, k) \prod_{j=1}^{V} \mathscr{V}_{\delta_{j}, n}^{(j)}(p, k),
$$

where $p$ are the loop momenta, $k$ are the external momenta, $\mathscr{P}_{-2, n}^{(i)}$ are the propagators, which have weighted degree -2 , and $\mathscr{V}_{\delta_{j}, n}^{(j)}$ are the vertices, with weighted degrees $\delta_{j}$. The integral measure $\mathrm{d}^{\widehat{d}} \widehat{p} \mathrm{~d}^{\bar{d}} \bar{p}$ is a weighted measure of degree $\mathrm{d} \equiv \widehat{d}+\bar{d} / n$, which we call "weighted dimensions". Performing a rescaling $(\widehat{k}, \bar{k}) \rightarrow\left(\lambda \widehat{k}, \lambda^{1 / n} \bar{k}\right)$, accompanied by an analogous change of variables $(\widehat{p}, \bar{p}) \rightarrow\left(\lambda \widehat{p}, \lambda^{1 / n} \bar{p}\right)$, and using the locality of counterterms, it is immediate to prove that the divergent part of $\mathscr{I}_{G}(k)$ is a weighted polynomial of degree

$$
\omega(G)=L đ-2 I+\sum_{j=1}^{V} \delta_{j}=L đ-2 I+\sum_{N, \alpha} \delta_{N}^{\alpha} v_{N}^{\alpha} .
$$

The usual relations

$$
L=I-V+1, \quad E+2 I=\sum_{N, \alpha} N v_{N}^{\alpha}
$$

allow us to write

$$
\omega(G)=d(E)+\sum_{N, \alpha} v_{N}^{\alpha}\left[\delta_{N}^{\alpha}-d(N)\right]
$$

where

$$
d(N) \equiv \mathrm{đ}\left(1-\frac{N}{2}\right)+N
$$

Observe that formula (2.5) reads exactly as the Lorentz invariant one, with the spacetime dimension replaced by đ. Even more, all considerations that follows can be obtained from the usual ones, operating this replacement. In practice, Lorentz violating quantum field theory lives, at high energies, in a reduced, generically fractional, dimension đ.

The theory is renormalizable if $\delta_{N}^{\alpha} \leq d(N)$ for every $N$ and $\alpha$. Indeed in that case counterterms satisfy the same inequality:

$$
\omega(G) \leq d(E) .
$$

Similarly, the theory is: ii) super-renormalizable, if $\delta_{N}^{\alpha}<d(N)$ for every $N$ and $\alpha$; iii) strictlyrenormalizable, if $\delta_{N}^{\alpha}=d(N)$ for every $N$ and $\alpha$; $\left.i v\right)$ non-renormalizable, if there exist vertices $N, \alpha$ with $\delta_{N}^{\alpha}>d(N)$.

The vertices with $\delta_{N}^{\alpha}=d(N)$ are called "weighted marginal", those with $\delta_{N}^{\alpha}<d(N)$ are called "weighted relevant" and those with $\delta_{N}^{\alpha}>d(N)$ are called "weighted irrelevant".

Counterterms have the form

$$
\left[\partial^{\omega(G)} \varphi^{E}\right] \prod_{N, \alpha} \lambda_{N, \alpha}^{v_{N}^{\alpha}} g^{v_{N}^{\alpha}(N-2)}=\left[\partial^{\omega(G)} \varphi^{E}\right] g^{E+2(L-1)} \prod_{N, \alpha} \lambda_{N, \alpha}^{v_{N}^{\alpha}}
$$


having used (2.4) again. If the theory is super-renormalizable, we have $\kappa \equiv[g]>0$. Then we have the stricter inequalities

$$
\delta_{N}^{\alpha} \leq d(N)-\kappa(N-2)
$$

and

$$
\omega(G) \leq d(E)-\kappa(E-2+2 L) .
$$

As promised, a renormalizable theory does not contain higher time derivatives. Indeed, by locality $\delta_{N}^{\alpha}$ cannot be negative, so (2.8) gives

$$
(đ+2 \kappa-2) N \leq 2(đ+2 \kappa) .
$$

By polynomiality, there must exist a finite, maximal $N$, therefore

$$
\text { đ }>2-2 \kappa .
$$

Then, (2.8) and (2.9) imply also $\delta_{N}^{\alpha} \leq 2, \omega(G) \leq 2$ (for $N \geq 2$ ). Higher time derivatives would violate such bounds.

The strictly renormalizable models have the $\delta_{N}^{(\alpha)}=d(N), \kappa=0$. Their lagrangian has the form

$$
\mathscr{L}_{(\widehat{d}, \bar{d})}=\frac{1}{2}(\widehat{\partial} \varphi)^{2}+\frac{1}{2 \Lambda_{L}^{2 n-2}}\left(\bar{\partial}^{n} \varphi\right)^{2}+\sum_{(N, \alpha)} \frac{\lambda_{(N, \alpha)}}{N ! \Lambda_{L}^{N(d / 2-1)+n d(N)-d}}\left[\bar{\partial}^{n d(N)} \varphi^{N}\right]_{\alpha} .
$$

We call the models (2.10) homogeneous. Homogeneity is preserved by renormalization, in the sense that there exists a subtraction scheme in which no lagrangian terms of weighted degrees smaller than $d(N)$ are turned on by renormalization. This fact is evident using the dimensionalregularization technique. Indeed, when $\delta_{N}^{(\alpha)}=d(N)$, the equality in (2.7) holds, so $\omega(G)=d(E)=$ $\delta_{E}^{(\alpha)}$.

The weighted scale invariance is anomalous at the quantum level. The weighted trace anomaly and its relation with the renormalization group are studied in [17]. The RG flow is more precisely understood as a "weighted RG flow", defined by the weights of the fields and couplings rather than by their dimensions. In particular, the infrared limit is the limit where $\Lambda_{L}$ is kept fixed, while the weightful parameters (including the RG scale $\mu$, which has weight 1 ) tend to infinity. Analogously, the ultraviolet limit is defined as the limit where $\Lambda_{L}$ is kept fixed, while the weightful parameters tend to zero. As a consequence, the fixed points of the weighted RG flow do depend on $\Lambda_{L}$.

Examples We begin with the $\varphi^{4}$-theories in four weighted dimensions. A simple Lorentzviolating solution is the model with $n=2$ described by the lagrangian

$$
\mathscr{L}_{(2,4)}=\frac{1}{2}(\widehat{\partial} \varphi)^{2}+\frac{1}{2 \Lambda_{L}^{2}}(\bar{\triangle} \varphi)^{2}+\frac{\lambda}{4 ! \Lambda_{L}^{2}} \varphi^{4}
$$

in six dimensions, with $\widehat{d}=2, \bar{d}=4$.

More generally, we have the family of 2(n+1)-dimensional theories

$$
\mathscr{L}_{(2,2 n)}=\frac{1}{2}(\widehat{\partial} \varphi)^{2}+\frac{1}{2 \Lambda_{L}^{2(n-1)}}\left(\bar{\partial}^{n} \varphi\right)^{2}+\frac{\lambda}{4 ! \Lambda_{L}^{2(n-1)}} \varphi^{4} .
$$


In general, for every Lorentz-invariant renormalizable theory there exists an infinite family of Lorentz-violating renormalizable theories.

In four dimensions the spacetime manifold can be split as $(\widehat{d}, \bar{d})=(0,4),(1,3),(2,2),(3,1)$, $(4,0)$. There is no non-trivial solution with $\widehat{d}=0$. For $\widehat{d}=1$ the only non-trivial solution has $n=2$ and reads

$$
\mathscr{L}_{(1,3)}=\frac{1}{2}(\widehat{\partial} \varphi)^{2}+\frac{1}{2 \Lambda_{L}^{2}}(\bar{\triangle} \varphi)^{2}+\frac{\lambda_{6}}{6 ! \Lambda_{L}^{4}} \varphi^{4}(\bar{\partial} \varphi)^{2}+\frac{\lambda_{10}}{10 ! \Lambda_{L}^{6}} \varphi^{10}
$$

For $\widehat{d}=2$ every integer $n>1$ gives a non-trivial solution. The simplest example is $(\widehat{d}, \bar{d})=(2,2)$, $n=2$, with lagrangian

$$
\mathscr{L}_{(2,2)}=\frac{1}{2}(\widehat{\partial} \varphi)^{2}+\frac{1}{2 \Lambda_{L}^{2}}(\bar{\triangle} \varphi)^{2}+\frac{\lambda_{4}}{4 ! \Lambda_{L}^{2}} \varphi^{2}(\bar{\partial} \varphi)^{2}+\frac{\lambda_{6}}{6 ! \Lambda_{L}^{2}} \varphi^{6}
$$

This model belongs to a family of $d=3,(2+n)$-dimensional $\varphi^{6}$-theories, whose lagrangian is

$$
\mathscr{L}_{(2, n)}=\frac{1}{2}(\widehat{\partial} \varphi)^{2}+\frac{1}{2 \Lambda_{L}^{2(n-1)}}\left(\bar{\partial}^{n} \varphi\right)^{2}+\frac{\lambda_{6}}{6 ! \Lambda_{L}^{2(n-1)}} \varphi^{6}
$$

when $n$ is odd and

$$
\mathscr{L}_{(2, n)}=\frac{1}{2}(\widehat{\partial} \varphi)^{2}+\frac{1}{2 \Lambda_{L}^{2(n-1)}}\left(\bar{\partial}^{n} \varphi\right)^{2}+\frac{1}{4 ! \Lambda_{L}^{2(n-1)}} \sum_{\alpha} \lambda_{\alpha}\left[\bar{\partial}^{n} \varphi^{4}\right]_{\alpha}+\frac{\lambda_{6}}{6 ! \Lambda_{L}^{2(n-1)}} \varphi^{6},
$$

when $n$ is even. Observe that (2.15) includes the Lorentz-invariant $\varphi^{6}$-theory in three spacetime dimensions, which is the case $n=1$.

For $\widehat{d}=3$ we have only $\varphi^{4}$ models (ignoring unstable $\varphi^{5}$ theories), for example

$$
\mathscr{L}_{(3,1)}^{\text {even }}=\frac{1}{2}(\widehat{\partial} \varphi)^{2}+\frac{1}{2 \Lambda_{L}^{4}}(\bar{\partial} \triangle \varphi)^{2}+\frac{\lambda_{4}}{4 ! \Lambda_{L}^{2}} \varphi^{2}(\bar{\partial} \varphi)^{2}
$$

with $n=3$ and the symmetry $\varphi \rightarrow-\varphi$. For $\widehat{d}=4$ we get again the Lorentz-invariant $\varphi^{4}$-theory.

Non-homogeneous models Non-homogeneous theories can be obtained from the homogeneous ones adding super-renormalizable terms, which are those that satisfy the strict inequality $\delta_{N}^{(\alpha)}<$ $d(N)$. For example, keeping the symmetry $\varphi \rightarrow-\varphi$, the non-homogeneous extension of (2.11) is just

$$
\mathscr{L}_{(2,4)}^{\mathrm{nh}}=\frac{1}{2}(\widehat{\partial} \varphi)^{2}+\frac{a}{2}(\bar{\partial} \varphi)^{2}+\frac{m^{2}}{2} \varphi^{2}+\frac{1}{2 \Lambda_{L}^{2}}(\bar{\triangle} \varphi)^{2}+\frac{\lambda}{4 ! \Lambda_{L}^{2}} \varphi^{4}
$$

and the one of (2.14) is

$$
\mathscr{L}_{(2,2)}^{\mathrm{nh}}=\frac{1}{2}(\widehat{\partial} \varphi)^{2}+\frac{a}{2}(\bar{\partial} \varphi)^{2}+\frac{m^{2}}{2} \varphi^{2}+\frac{1}{2 \Lambda_{L}^{2}}(\bar{\triangle} \varphi)^{2}+\frac{\lambda_{4}}{4 ! \Lambda_{L}^{2}} \varphi^{2}(\bar{\partial} \varphi)^{2}+\frac{\lambda_{4}^{\prime}}{4 !} \varphi^{4}+\frac{\lambda_{6}}{6 ! \Lambda_{L}^{2}} \varphi^{6}
$$


Fermions The considerations just recalled are easily generalized to fermions. The weight of a fermion field is $(\mathrm{d}-1) / 2$. Renormalizable theories are those that contain all vertices and quadratic terms with weight not larger than đ and only those. Nontrivial stable renormalizable theories containing only fermions exist for $\mathrm{d} \leq 2$.

The quadratic lagrangian reads

$$
\mathscr{L}_{\text {free }}=\bar{\psi} \widehat{\partial} \psi+\sum_{j=0}^{n} \frac{a_{j}}{\Lambda_{L}^{j-1}} \bar{\psi} \bar{\partial}^{j} \psi
$$

and its propagator is

$$
\frac{-i \not \vec{p}+\sum_{j=0}^{n}(-i)^{j} a_{j} \frac{\not p^{j}}{\Lambda_{L}^{j-1}}}{\widehat{p}^{2}+\sum_{j=0}^{n}(-1)^{j} b_{j} \frac{\left(\bar{p}^{2}\right)^{j}}{\Lambda_{L}^{j-2}}} \quad b_{j}=\sum_{i=0}^{\min (2 j, n)}(-1)^{i} a_{j} a_{2 j-i} .
$$

We can normalize $a_{n}$ to 1 , while $a_{0}$ is the mass $m$. As before, for the purposes of renormalization we can treat the terms with fewer than $n \bar{\partial}$-derivatives as vertices, and use the homogeneous propagator

$$
\frac{-i \widehat{p}+(-i)^{n} \frac{\not^{n}}{\Lambda_{L}^{n-1}}}{\widehat{p}^{2}+\frac{\left(\bar{p}^{2}\right)^{n}}{\Lambda_{L}^{2-2}}}
$$

Label the vertices that have $2 N \psi-\bar{\psi}$-legs by means of indices $\alpha$. Call $\delta_{N}^{\alpha}$ the weighted degree of the derivatives acting on them. Consider a diagram $G$ with $2 E$ external $\psi-\bar{\psi}$-legs, constructed with $v_{N}^{\alpha}$ vertices of type $N, \alpha$. Once the subdivergences have been subtracted away, its overall divergence is a weighted polynomial of degree

$$
\omega(G)=d-E(d-1)+\sum_{N, \alpha} v_{N}^{\alpha}\left[\delta_{N}^{\alpha}-d_{F}(N)\right]
$$

in the external momenta, where

$$
d_{F}(N) \equiv đ(1-N)+N
$$

Renormalizability demands

$$
\delta_{N}^{\alpha} \leq d_{F}(N)
$$

Pure fermionic homogeneous models have strictly renormalizable vertices, namely those with $\delta_{N}^{\alpha}=$ $d_{F}(N)$. Their lagrangian has the form

$$
\mathscr{L}=\bar{\psi} \widehat{\partial} \psi+\frac{1}{\Lambda_{L}^{n-1}} \bar{\psi}^{n} \bar{\partial}^{n} \psi+\sum_{N, \alpha} \frac{\lambda_{N, \alpha}}{(N !)^{2} \Lambda_{L}^{(n-1)(N-\widehat{d}-N \widehat{d})}}\left[\bar{\partial}^{n d_{F}(N)} \bar{\psi}^{N} \psi^{N}\right]_{\alpha} .
$$

Here $\left[\bar{\partial}^{n d_{F}(N)} \bar{\psi}^{N} \psi^{N}\right]_{\alpha}$ denotes a basis of lagrangian terms constructed with $N$ fields $\psi, N$ fields $\bar{\psi}$ and $n d_{F}(N) \bar{\partial}$-derivatives, invariant under the reduced Lorentz symmetry. For simplicity, we can assume also invariance under parities in both portions of spacetime. 
Let us concentrate on four spacetime dimensions. The Lorentz split $(1,3)$ gives admits infinitely many non-trivial solutions, beginning from $n=3$. For example, the $n=3$ and $n=6$ theories read

$$
\begin{aligned}
& \mathscr{L}_{(1,3)}=\bar{\psi} \widehat{\partial} \psi+\frac{1}{\Lambda_{L}^{2}} \bar{\psi} \bar{\Delta} \bar{\partial} \psi+\sum_{\alpha} \frac{\lambda_{\alpha}}{\Lambda_{L}^{2}}\left[\bar{\psi}^{2} \psi^{2}\right]_{\alpha}, \\
& \mathscr{L}_{(1,3)}^{\prime}=\bar{\psi} \widehat{\partial} \psi+\frac{1}{\Lambda_{L}^{5}} \bar{\psi} \bar{\Delta}^{3} \psi+\sum_{\alpha} \frac{\lambda_{\alpha}}{\Lambda_{L}^{5}}\left[\bar{\partial}^{3} \bar{\psi}^{2} \psi^{2}\right]_{\alpha}+\sum_{\alpha} \frac{\lambda_{\alpha}^{\prime}}{\Lambda_{L}^{5}}\left[\bar{\psi}^{3} \psi^{3}\right]_{\alpha},
\end{aligned}
$$

respectively. The Lorentz splits $(2,2)$ and $(3,1)$ do not admit non-trivial solutions.

Now we study the models containing coupled scalars and fermions. It is important to note that when different types of fields are involved, they must have the same $n$. We classify the vertices with labels $N_{\psi}, N_{\varphi}, \alpha$, where $2 N_{\psi}$ is the number of $\psi$ - $\bar{\psi}$-legs, $N_{\varphi}$ is the number of $\varphi$-legs and $\alpha$ is an extra label that distinguishes vertices with different structures. Call $\delta_{N_{\psi}, N_{\varphi}}^{\alpha}$ the weighted degree of the $\alpha$-th vertex. Consider a diagram $G$ with $2 E_{\psi}$ external $\psi-\bar{\psi}$-legs, $E_{\varphi}$ external $\varphi$-legs and $v_{N_{\psi}, N_{\varphi}}^{\alpha}$ vertices of type $N_{\psi}, N_{\varphi}, \alpha$. Once the subdivergences have been subtracted away, the overall divergent part of $G$ a is a weighted polynomial of degree

$$
\begin{aligned}
\omega(G)= & \text { đ } \\
& +E_{\psi}(\mathrm{d}-1)-\frac{E_{\varphi}}{2}(\mathrm{~d}-2) \\
& \sum_{N_{\psi}, N_{\varphi}, \alpha} v_{N_{\psi}, N_{\varphi}}^{\alpha}\left[\delta_{N_{\psi}, N_{\varphi}}^{\alpha}-d\left(1-N_{\psi}-\frac{N_{\varphi}}{2}\right)-N_{\psi}-N_{\varphi}\right]
\end{aligned}
$$

in the external momenta. Renormalizability demands

$$
\delta_{N_{\psi}, N_{\varphi}}^{\alpha} \leq đ\left(1-N_{\psi}-\frac{N_{\varphi}}{2}\right)+N_{\psi}+N_{\varphi} \equiv d\left(N_{\psi}, N_{\varphi}\right) .
$$

Because $\delta_{N_{\psi}, N_{\varphi}}^{\alpha}$ is non-negative, the numbers of fermionic and bosonic legs are bound by the inequality

$$
N_{\psi}(\text { đ }-1)+\frac{N_{\varphi}}{2}(\text { đ }-2) \leq \text { đ }
$$

The homogeneous models have a lagrangian of the form

$$
\begin{aligned}
\mathscr{L}= & \bar{\psi} \widehat{\partial} \psi+\frac{\eta}{\Lambda_{L}^{n-1}} \bar{\psi}^{n} \psi+\frac{1}{2}(\widehat{\partial} \varphi)^{2}+\frac{1}{2 \Lambda_{L}^{2 n-2}}\left(\bar{\partial}^{n} \varphi\right)^{2} \\
& +\sum_{N_{\psi}, N_{\varphi}, \alpha} \frac{\lambda_{N_{\psi}, N_{\varphi}, \alpha}}{N_{\varphi} !\left(N_{\psi} !\right)^{2} \Lambda_{L}^{(n-1)\left(N_{\varphi}+N_{\psi}+\widehat{d}-\widehat{d} N_{\psi}-\widehat{d} N_{\varphi} / 2\right)}}\left[\bar{\partial}^{n d\left(N_{\psi}, N_{\varphi}\right)} \bar{\psi}^{N_{\psi}} \psi^{N_{\psi}} \varphi^{N_{\varphi}}\right]_{\alpha} .
\end{aligned}
$$

In four dimensions the splitting $(1,3)$ has a unique non-trivial solution, which is the model $(2.13)$ coupled to fermions. It has $n=2$ and its lagrangian reads

$$
\begin{aligned}
\mathscr{L}_{(1,3)}= & \bar{\psi} \widehat{\partial} \psi+\frac{\eta}{\Lambda_{L}} \bar{\psi} \bar{\Delta} \psi+\frac{1}{2}(\widehat{\partial} \varphi)^{2}+\frac{1}{2 \Lambda_{L}^{2}}(\bar{\Delta} \varphi)^{2}+\frac{\lambda_{2}}{2 \Lambda_{L}^{2}} \varphi^{2}(\bar{\psi} \overleftrightarrow{\bar{\partial}} \psi)+\frac{\lambda_{2}^{\prime}}{2 \Lambda_{L}^{2}} \varphi^{2} \bar{\partial} \cdot(\overline{\psi \gamma} \psi) \\
& +\frac{\lambda_{4}}{4 ! \Lambda_{L}^{3}} \varphi^{4} \bar{\psi} \psi+\frac{\lambda_{6}}{6 ! \Lambda_{L}^{4}} \varphi^{4}(\bar{\partial} \varphi)^{2}+\frac{\lambda_{10}}{10 ! \Lambda_{L}^{6}} \varphi^{10} .
\end{aligned}
$$


The splitting $(2,2)$ admits infinitely many solutions. The simplest one is the theory with $n=2$, symmetric under $\varphi \leftrightarrow-\varphi$, that couples (2.14) to fermions:

$$
\mathscr{L}_{(2,2)}=\bar{\psi} \widehat{\partial} \psi+\frac{\eta}{\Lambda_{L}} \bar{\psi} \bar{\psi} \psi+\frac{1}{2}(\widehat{\partial} \varphi)^{2}+\frac{1}{2 \Lambda_{L}^{2}}(\bar{\Delta} \varphi)^{2}+\frac{\lambda_{2}}{2 \Lambda_{L}} \varphi^{2} \bar{\psi} \psi+\frac{\lambda_{4}}{4 ! \Lambda_{L}^{2}} \varphi^{2}(\bar{\partial} \varphi)^{2}+\frac{\lambda_{6}}{6 ! \Lambda_{L}^{2}} \varphi^{6},
$$

The splitting $(3,1)$ admits, again, infinitely many solutions.

The simplest homogeneous examples are the $₫=2$, four-fermion models

$$
\mathscr{L}_{\mathrm{d}=2}=\bar{\psi}\left(\widehat{\partial}+\frac{\bar{\partial}^{n}}{\Lambda_{L}^{n-1}}\right) \psi-\frac{\lambda^{2}}{2 \Lambda_{L}^{d-2}}(\bar{\psi} \psi)^{2} .
$$

Stable coupled scalar and fermion theories exist for $₫ \leq 4$.

Gauge fields The gauge field $A_{\mu}=A_{\mu}^{a} T^{a}$, with $T^{a}$ anti-Hermitian, is decomposed as $A=(\widehat{A}, \bar{A})$. The covariant derivative

$$
D=(\widehat{D}, \bar{D})=(\widehat{\partial}+g \widehat{A}, \bar{\partial}+g \bar{A})
$$

induces the weight assignments

$$
[g \widehat{A}]=[\widehat{D}]=1, \quad[g \bar{A}]=[\bar{D}]=\frac{1}{n},
$$

where $g$ is the gauge coupling. An important fact is that Lorentz violating gauge theories admit only the spacetime splitting into space and time. Theories with $\widehat{d}>1$ have additional spurious divergences that cannot be eliminated [28, 29]. The field strength is split as

$$
\tilde{F}_{\mu v} \equiv F_{\widehat{\mu} \bar{v}}, \quad \bar{F}_{\mu \nu} \equiv F_{\overline{\mu \nu}} .
$$

( $F_{\widehat{\mu} \widehat{v}}$ is absent at $\left.\widehat{d}=1\right)$.

In Lorentz violating gauge theories the BRST symmetry is the same as usual,

$$
\begin{aligned}
& s A_{\mu}^{a}=D_{\mu}^{a b} C^{b}=\partial_{\mu} C^{a}+g f^{a b c} A_{\mu}^{b} C^{c}, \quad s C^{a}=-\frac{g}{2} f^{a b c} C^{b} C^{c}, \\
& s \bar{C}^{a}=B^{a}, \quad s B^{a}=0, \quad s \psi^{i}=-g T_{i j}^{a} C^{a} \psi^{j},
\end{aligned}
$$

etc., where $B^{a}$ are Lagrange multipliers for the gauge-fixing. The quadratic gauge field lagrangian reads

$$
\mathscr{L}_{Q}=\frac{1}{2} F_{\widehat{\mu} \bar{\nu}}^{2}-\frac{1}{4} F_{\overline{\mu \nu}} \tau(\bar{\Upsilon}) F_{\overline{\mu \nu}}
$$

where $\tau$ is a polynomial of degree $n-1$ and $\bar{\Upsilon}=-\bar{D}^{2} / \Lambda_{L}^{2}$. The most convenient gauge-fixing is

$$
\mathscr{L}_{\mathrm{gf}}=s \Psi, \quad \Psi=\bar{C}^{a}\left(-\frac{\lambda}{2} B^{a}+\mathscr{G}^{a}\right), \quad \mathscr{G}^{a} \equiv \widehat{\partial} \cdot \widehat{A}^{a}+\zeta(\bar{v}) \bar{\partial} \cdot \bar{A}^{a},
$$

where $\bar{v} \equiv-\bar{\partial}^{2} / \Lambda_{L}^{2}, \zeta$ is a polynomial of degree $n-1$ and $\lambda$ can be a constant, but also a function of $\bar{v}$. The total gauge-fixed action is

$$
\mathscr{S}=\int \mathrm{d}^{d} x\left(\mathscr{L}_{Q}+\mathscr{L}_{\mathrm{I}}+\mathscr{L}_{\mathrm{gf}}\right)
$$


where $\mathscr{L}_{I}$ collects the terms that are at least cubic in the field strength.

The gauge-field propagator can be worked out from the free subsector of (2.23), after integrating $B^{a}$ out, which amounts to add $\left(\mathscr{G}^{a}\right)^{2} /(2 \lambda)$ to the quadratic lagrangian $\mathscr{L}_{Q}$. In the "Feynman" gauge

$$
\zeta=\lambda=\tau
$$

we find a nice diagonal propagator

$$
\left(\begin{array}{cc}
\langle\widehat{A} \widehat{A}\rangle & \langle\widehat{A A}\rangle \\
\langle\bar{A} \widehat{A}\rangle & \langle\overline{A A}\rangle
\end{array}\right)=\frac{1}{\widehat{k}^{2}+\tau \bar{k}^{2}}\left(\begin{array}{ll}
\tau & 0 \\
0 & \bar{\delta}
\end{array}\right),
$$

where now $\tau$ and $\zeta$ are functions of $\bar{k}^{2} / \Lambda_{L}^{2}$. The ghost propagator is $1 /\left(\widehat{k}^{2}+\tau \bar{k}^{2}\right)$. Observe that in the gauge (2.24) integrating $B$ out amounts to add the non-local term

$$
\frac{1}{2}\left(\mathscr{G}^{a}\right) \frac{1}{\tau}\left(\mathscr{G}^{a}\right)
$$

to $\mathscr{L}_{Q}$. This is legitimate, since the action (2.23) is local before integrating $B$ out, and $B$ is nonpropagating (see (2.22)).

The consistency of (2.21) is explained by a simple weight assignment. The kinetic lagrangian $\mathscr{L}_{Q}$ contains $\tilde{F}^{2}$, so $\tilde{F}$ must have weight đ/2. Since $[\tilde{F}]=[\bar{\partial}]+[\widehat{A}]=[\widehat{\partial}]+[\bar{A}]$, we have

$$
[\widehat{A}]=\frac{đ}{2}-\frac{1}{n}, \quad[\bar{A}]=\frac{đ}{2}-1, \quad[\tilde{F}]=\frac{đ}{2}, \quad[\bar{F}]=\frac{đ}{2}-1+\frac{1}{n} .
$$

The weight of the gauge coupling is

$$
[g]=1+\frac{1}{n}-\frac{đ}{2}
$$

Observe that $[g]>0$ in four dimensions, for $n>1$, where gauge interactions are always superrenormalizable. We also find $[\zeta]=[\lambda]=[\tau]=2-2 / n$.

The quadratic terms of the ghost Lagrangian contain $\bar{C} \widehat{\partial}^{2} C$ and $\lambda B^{2}$, which have weight đ, so we have the weight assignments

$$
[C]=[\bar{C}]=\frac{\text { đ }}{2}-1, \quad[s]=\frac{1}{n}, \quad[B]=\frac{\text { đ }}{2}-1+\frac{1}{n} .
$$

\section{Standard-Extended models}

Now we are ready to present our CPT invariant Lorentz violating extensions of the Standard Model [20, 23]. In four dimensions we have $\mathbb{d}=1+3 / n$. The simplest choice is $n=3$, which means weighted dimension 2. The lagrangian reads, in Minkowski space,

$$
\begin{gathered}
\mathscr{L}=\mathscr{L}_{Q}+\mathscr{L}_{\text {kin } f}+\mathscr{L}_{H}+\mathscr{L}_{Y}-\frac{\bar{g}^{2}}{4 \Lambda_{L}}(L H)^{2}-\sum_{I=1}^{5} \frac{1}{\Lambda_{L}^{2}} g \overline{D F}\left(\bar{\chi}_{I} \bar{\gamma} \chi_{I}\right)+\frac{Y_{f}}{\Lambda_{L}^{2}} \bar{\psi} \psi \bar{\psi} \psi-\frac{g}{\Lambda_{L}^{2}} \bar{F}^{3} \\
-\frac{1}{\Lambda_{L}^{2}} g \bar{g} \bar{\psi} \psi \bar{F} H-\frac{1}{\Lambda_{L}^{2}}\left(\bar{g}^{3} \bar{\psi} \psi H^{3}+\bar{g}^{2} \bar{\psi} \bar{D} \psi H^{2}+\bar{g} \bar{\psi} \bar{D}^{2} \psi H\right)-\frac{1}{\Lambda_{L}^{4}}\left(g \bar{D}^{2} \bar{F}+g^{2} \bar{F}^{2}\right) H^{\dagger} H,(3.1)
\end{gathered}
$$


where

$$
\begin{aligned}
\mathscr{L}_{Q}= & \frac{1}{4} \sum_{G}\left(2 F_{\widehat{\mu} \bar{v}}^{G} F_{\widehat{\mu} \bar{v}}^{G}-F_{\overline{\mu \nu}}^{G} \tau^{G}(\bar{\Upsilon}) F_{\overline{\mu \nu}}^{G}\right) \\
\mathscr{L}_{\text {kin } f=} & \sum_{a, b=1}^{3} \sum_{I=1}^{5} \bar{\chi}_{I}^{a} i\left(\delta^{a b} \widehat{\bar{D}}-\frac{b_{0}^{I a b}}{\Lambda_{L}^{2}} \bar{D}^{3}+b_{1}^{I a b} \bar{D}\right) \chi_{I}^{b}, \\
\mathscr{L}_{H}= & \left|\widehat{D}_{\widehat{\mu}} H\right|^{2}-\frac{a_{0}}{\Lambda_{L}^{4}}\left|\bar{D}^{2} \bar{D}_{\bar{\mu}} H\right|^{2}-\frac{a_{1}}{\Lambda_{L}^{2}}\left|\bar{D}^{2} H\right|^{2}-a_{2}\left|\bar{D}_{\bar{\mu}} H\right|^{2}-\mu_{H}^{2}|H|^{2}-\frac{\lambda_{4} \bar{g}^{2}}{4}|H|^{4}-\frac{\lambda_{6} \bar{g}^{4}}{36 \Lambda_{L}^{2}}|H|^{6} \\
& -\frac{\lambda_{4}^{(3)} \bar{g}^{2}}{4 \Lambda_{L}^{2}}|H|^{2}\left|\bar{D}_{\bar{\mu}} H\right|^{2}-\frac{\lambda_{4}^{(2)} \bar{g}^{2}}{4 \Lambda_{L}^{2}}\left|H^{\dagger} \bar{D}_{\bar{\mu}} H\right|^{2}-\frac{\bar{g}^{2}}{4 \Lambda_{L}^{2}}\left[\lambda_{4}^{(1)}\left(H^{\dagger} \bar{D}_{\bar{\mu}} H\right)^{2}+\text { h.c. }\right] \\
\mathscr{L}_{Y}= & -\bar{g} \Omega_{i} H^{i}+\text { h.c., } \quad \Omega_{i}=\sum_{a, b=1}^{3} Y_{1}^{a b} \bar{L}^{a i} \ell_{R}^{b}+Y_{2}^{a b} \bar{u}_{R}^{a} Q_{L}^{b j} \varepsilon^{j i}+Y_{3}^{a b} \bar{Q}_{L}^{a i} d_{R}^{b},
\end{aligned}
$$

$i, j$ are $S U(2)_{L}$ indices, $\chi_{1}^{a}=L^{a}=\left(v_{L}^{a}, \ell_{L}^{a}\right), \chi_{2}^{a}=Q_{L}^{a}=\left(u_{L}^{a}, d_{L}^{a}\right), \chi_{3}^{a}=\ell_{R}^{a}, \chi_{4}^{a}=u_{R}^{a}$ and $\chi_{5}^{a}=d_{R}^{a}$. Moreover, $v^{a}=\left(v_{e}, v_{\mu}, v_{\tau}\right), \ell^{a}=(e, \mu, \tau), u^{a}=(u, c, t)$ and $d^{a}=(d, s, b)$. The sum $\sum_{G}$ is over the gauge groups $S U(3)_{c}, S U(2)_{L}$ and $U(1)_{Y}$. Finally, $\tau^{G}$ are polynomials of degree 2 . Gauge anomalies cancel out exactly as in the Standard Model [20]. The "boundary conditions" such that Lorentz invariance is recovered at low energies are that $b_{1}^{I a b}$ tend to $\delta^{a b}$ and $a_{2}, \tau^{G}$ tend to 1 (one such condition can be fulfilled normalizing the space coordinates $\bar{x}$ ).

The gauge coupling $g$ has weight $1 / 3$. Since the weight of a scalar field vanishes in $\mathrm{d}=2 \mathrm{a}$ constant $\bar{g}$ of weight $1 / 3$ is attached to the scalar legs to ensure renormalizability. The weights of all other parameters are determined so that each lagrangian term has weight $2(=đ)$. We have neutrino masses $\sim v^{2} / \Lambda_{L}, v$ being the Higgs vev, assuming that all other parameters involved in the vertex $(L H)^{2} / \Lambda_{L}$ are of order 1 . If this is the origin of neutrino masses, reasonable estimates of their values (a fraction of $\mathrm{eV}$ ) give $\Lambda_{L} \sim 10^{14} \mathrm{GeV}$.

By covariance, the coupling $\bar{g}$ attached to the scalar legs must satisfy $[\bar{g}] \leq[g][29]$, so we can choose $[\bar{g}]=1 / 3$. Then the most general lagrangian is (3.1) plus the extra terms

$$
\begin{aligned}
& \bar{g}^{6} H^{8}, \quad \bar{g}^{4} \bar{D}^{2} H^{6}, \quad \bar{g}^{2} \bar{D}^{4} H^{4}, \quad g \bar{g}^{2} \bar{D}^{2} \bar{F} H^{4}, \quad g \bar{D}^{4} \bar{F} H^{2}, \quad g^{2} \bar{g}^{2} \bar{F}^{2} H^{4}, \quad g^{2} \bar{D}^{2} \bar{F}^{2} H^{2}, \quad g^{3} \bar{F}^{3} H^{2}, \\
& g^{2} \bar{F}^{4}, \quad g \bar{D}^{2} \bar{F}^{3}, \quad g \bar{\varepsilon} \tilde{F} \bar{D}^{2} H^{2}, \quad g^{2} \bar{\varepsilon} \tilde{F} \bar{F} H^{2}, \quad \bar{\varepsilon} \tilde{F} \bar{D}^{2} \bar{F}, \quad g \bar{\varepsilon} \bar{F} \tilde{F} \bar{F}, \quad \bar{\varepsilon} \bar{F} \widehat{D} \overline{D F},
\end{aligned}
$$

and those obtained suppressing some fields and/or derivatives, where $\bar{\varepsilon}$ is the $\varepsilon$-tensor with three space indices. The extra terms (3.3) can be consistently dropped, because they are not generated back by renormalization.

Scalarless model Our scalarless Standard-Extended Model is obtained suppressing the Higgs field in (3.1) and reads

$$
\mathscr{L}_{\text {noH }}=\mathscr{L}_{Q}+\mathscr{L}_{\text {kin } f}-\sum_{I=1}^{5} \frac{1}{\Lambda_{L}^{2}} g \overline{D F}\left(\bar{\chi}_{I} \bar{\gamma} \chi_{I}\right)+\frac{Y_{f}}{\Lambda_{L}^{2}} \bar{\psi} \psi \bar{\psi} \psi-\frac{g}{\Lambda_{L}^{2}} \bar{F}^{3} .
$$

Obviously, gauge anomalies still cancel. We see that the simplification with respect to (3.1) is considerable. 
At energies $\gg \Lambda_{L}$ gauge and Higgs fields become free and decouple, because their interactions are super-renormalizable. Both theories (3.1) and (3.4) become a four fermion model in two weighted dimensions, with lagrangian

$$
\mathscr{L}_{4 \mathrm{f}}=\sum_{a, b=1}^{3} \sum_{I=1}^{5} \bar{\chi}_{I}^{a} i\left(\delta^{a b} \widehat{\not}-\frac{b_{0}^{I a b}}{\Lambda_{L}^{2}} \bar{\partial}^{3}+b_{1}^{I a b} \bar{\not}\right) \chi_{I}^{b}+\frac{Y_{f}}{\Lambda_{L}^{2}} \bar{\psi} \psi \bar{\psi} \psi .
$$

We have kept also the terms multiplied by $b_{1}^{\text {Iab }}$, since they are necessary to recover Lorentz invariance at low energies.

The model (3.4) can describe the known low-energy physics, including the Higgs boson as a composite field, by means of a dynamical symmetry breaking mechanism triggered by the four fermion vertices, where some quark-antiquark bilinears acquire expectation values [23]. At energy scales much smaller than $\Lambda_{L}$ the low-energy effective theory resembles a Standard Model with one or more Higgs doublets. However, the masses of composite Higgs bosons, as well as their selfcouplings and couplings to quarks and gauge fields, are not free, but unambiguously determined by (3.4). This is a crucial difference with respect to the ordinary Nambu-Jona-Lasinio framework [22], which was studied in $[30,31]$ and makes use of non-renormalizable interactions. The predictivity of the ordinary approach was questioned in ref. [32], where it was shown that the unknown highenergy physics, duly parametrized, can add enough extra parameters to the low-energy effective action, and make it completely equivalent to the Standard Model (with elementary Higgs field), equipped with all its free constants. The model 3.4, on the contrary, has an unambiguous highenergy behavior. With respect to other approaches to composite Higgs bosons, such as technicolor [36], or the introduction of extra heavy gauge bosons to renormalize four fermion vertices [35], it has the advantage of being conceptually more economic.

The dynamical symmetry breaking is a non-perturbative mechanism to generate low-energy effects from otherwise suppressed high-energy interactions. The dynamical symmetry breaking might reverberate the Lorentz violation down to low energies. If that happened, our scalarless model (3.4) would be in trouble. However, using the large $N_{c}$ expansion we can prove that Lorentz symmetry remains highly suppressed even when the dynamical symmetry breaking takes place.

Specifically, the dynamical symmetry mechanism produces fermion condensates $\langle\bar{q} q\rangle$. The effective potential, calculated in the large $N_{c}$ expansion, and has a Lorentz invariant (local) minimum, which gives masses to the fermions. Massive scalar bound states (composite Higgs bosons) emerge, together with Goldstone bosons [34]. Then gauge interactions are switched back on, and the Goldstone bosons associated with the breaking of $S U(2)_{L} \times U(1)_{Y}$ to $U(1)_{Q}$ are "eaten" by the $W^{ \pm}$and $Z$ bosons, which become massive. Curiously, the relation between $m_{t}$ and the Fermi constant,

$$
\frac{1}{G_{F}}=\frac{N_{c} m_{t}^{2}}{4 \pi^{2} \sqrt{2}} \ln \frac{\Lambda_{L}^{2}}{m_{t}^{2}} .
$$

turns out to be in astonishingly good agreement with the experiment: using our estimated value $\Lambda_{L}=10^{14} \mathrm{GeV}$, we find $m_{t}=171.6 \mathrm{GeV}$, when Particle Data Group gives $m_{t}=171.2 \pm 2.1 \mathrm{GeV}$.

\section{Conclusions}

Unitary Lorentz violating renormalizable quantum field theories in flat space can be obtained 
improving the UV behavior of propagators with the help of higher space derivatives. The removal of divergences is governed by a weighted power-counting criterion. If the lagrangian has the form dictated by this criterion, time derivatives are "protected", in the sense that no higher time derivatives are turned on by renormalization. When gauge fields are present, spacetime must be split into time and space. The Standard Model admits interesting Lorentz violating extensions. For example, there exist models that can renormalize two scalar-two fermion vertices, and therefore give masses to the (left-handed) neutrinos without the need to introduce right-handed neutrinos, nor other extra fields, and without violating CPT. We have found that the simplest model with such properties can contain also four fermion interactions, and therefore describe proton decay. The cancellation of anomalies is inherited from the one of the Standard Model.

Our extended models become very simple at high energies $\left(\gg \Lambda_{L}\right)$, where all gauge and Higgs interactions, being super-renormalizable, disappear. There survives a four fermion model in two weighted dimensions, which admits a dynamical symmetry breaking. In spite of the fact that such a four fermion model is Lorentz violating, the dynamically generated vacuum and the low energy effective action are Lorentz invariant. We have therefore focused on the scalarless extended model (3.4). In the large $N_{c}$ expansion the dynamical symmetry breaking generates composite massive Higgs bosons and gives masses to fermions and gauge bosons. The model is predictive, in the sense that it does not contain the ambiguities of previous approaches, which relied on the non-renormalizable Nambu-Jona-Lasinio mechanism, and is candidate to explain all low energy physics. The leading order of the large $N_{c}$ expansion, with gauge interactions switched off, does not allow us to make very precise quantitative predictions, although the relation (3.6) between the Fermi constant and the top mass turns out to be astonishingly right.

A step forward towards more precise predictions is to include the effects of the RG flow from energies $\sim m_{t}$ to $\Lambda_{L}$, and study the condition of compositeness at energies $\sim \Lambda_{L}$ [31]. However, in our Lorentz violating theories the RG flow is considerably different from the usual one: it coincides with the usual one at energies $\sim m_{t}$, since the low energy theory (with composite Higgs bosons included) is renormalizable by ordinary power counting; on the other hand, it changes completely as we move to energies $\sim \Lambda_{L}$, because there, gauge interactions do not run.

The dynamical symmetry breaking mechanism can of course take place also in the Higgsed model (3.1), if the four fermion vertices are chosen appropriately. There, its effects sum to those of the elementary Higgs doublet. It can also be applied to Standard Model extensions that contain new types of fermions, interacting by four fermion vertices, such as those considered in ref.s [37].

If we accept that Lorentz invariance is violated at high energies there remains to explain why it should be recovered at low energies, since generically renormalization make the couplings run independently. It is of course possible to restore Lorentz invariance at low energies by means of a fine tuning, which would be easier to justify if the Lorentz invariant surface were infrared stable.

\section{References}

[1] D. Colladay and V.A. Kostelecký, Lorentz-violating extension of the Standard Model, Phys. Rev. D58 (1998) 116002 and arXiv:hep-ph/9809521.

[2] V.A. Kostelecký, Ed., Proceedings of the Fourth Meeting on CPT and Lorentz Symmetry, World Scientific, Singapore, 2008; 
V.A. Kostelecký and N. Russell, Data tables for Lorentz and CTP violation, ibid. p. 308 and arXiv:0801.0287 [hep-ph].

[3] V.A. Rubakov and P.G. Tinyakov, Infrared-modified gravities and massive gravitons, Phys. Usp. 51 (1008) 759 and arXiv:0802.4379 [hep-th].

[4] V.A. Rubakov, Lorentz-violating graviton masses:getting around ghosts, low strong coupling scale and vDVZ discontinuity, arXiv:hep-th/047104.

[5] V.A. Kostelecký and S. Samuel, Spontaneous breaking of Lorentz symmetry in string theory, Phys. Rev. D 39 (1989) 683;

S.M. Carroll, G.B. Field and R. Jackiw, Limits on a Lorentz and Parity Violating Modification of Electrodynamics, Phys. Rev. D 41 (1990) 1231;

G. Amelino-Camelia, J.R. Ellis, N.E. Mavromatos, D.V. Nanopoulos, S. Sarkar, Tests of quantum gravity from observations of gamma-ray bursts, Nature 393 (1998) 763 and arXiv:astro-ph/9712103;

S.R. Coleman and G.L. Glashow, High-energy tests of Lorentz invariance, Phys. Rev. D59 (1999) 116008 and arXiv:hep-ph/9812418;

O. Bertolami and C.S. Carvalho, Proposed astrophysical test of Lorentz invariance, Phys. Rev. D 61 (2000) 103002 and arXiv:gr-qc/9912117;

J.R. Ellis, N.E. Mavromatos, D.V. Nanopoulos, A.S. Sakharov and E.K.G. Sarkisyan, Robust limits on Lorentz violation from gamma-ray bursts, Astropart. Phys. 25 (2006) 402, Astropart. Phys. 29 (2008) 158 (Erratum) and arXiv:astro-ph/05104172;

M. Rodriguez Martinez and T. Piran, Constraining Lorentz violations with gamma ray bursts, JCAP 0604 (2006) 006 and arXiv:astro-ph/0601219.

[6] R. Jackiw and V.A. Kostelecký, Radiatively induced Lorentz and CPT violation in electrodynamics, Phys. Rev. Lett. 82 (1999) 3572 and arXiv:hep-ph/9901358;

M. Perez-Victoria, Physical (ir)relevance of ambiguities to Lorentz and CPT violation in QED, JHEP 0104 (2001) 032 and arXiv:hep-th/0102021;

N.R. Bruno, G. Amelino-Camelia and J. Kowalski-Glikman, Deformed boost transformations that saturate at the Planck scale, Phys. Lett. B 522 (2001) 133 and arXiv:hep-th/0107039;

V.A. Kostelecký, Gravity, Lorentz violation, and the standard model, Phys. Rev. D 69 (2004) 105009 and arXiv:hep-th/0312310;

N. Arkani-Hamed, H.-C. Cheng, M. Luty and J. Thaler, Universal dynamics of spontaneous Lorentz violation and a new spin-dependent inverse-square law force, JHEP 0507 (2005) 029 and arXiv:hep-ph/0407034;

J. Gamboa, J. Lopez-Sarrion and A.P. Polychronakos, Ultraviolet modified photons and anisotropies in the cosmic microwave background radiation, Phys. Lett. B 634 (2006) 471 and arXiv:hep-ph/0510113;

A.G. Cohen and S.L. Glashow, Very special relativity, Phys. Rev. Lett. 97 (2006) 021601 and arXiv:hep-ph/0601236;

A.G. Cohen and S.L. Glashow, A Lorentz-violating origin of the neutrino mass?, arXiv:hep-ph/0605036;

V.A. Kostelecký and M. Mewes, Lorentz and CPT violations in neutrinos, Phys. Rev. D 69 (2004) 016005 and arXiv:hep-ph/0309025;

F. Klinkhamer, Lorentz-noninvariant neutrino oscillations: models and predictions, Int. J. Mod. Phys. A 21 (2006) 161 and arXiv:hep-ph/0407200. 
[7] See for example, D.B. Kaplan, M.J. Savage and M.B.Wise, Nucleon-nucleon scattering from effective field theory, Nucl. Phys. B478 (1996) 629 and arXiv:nucl-th/9605002.

[8] S. Weinberg, Phenomenological lagrangians, Physica A96 (1979) 327;

For a review, see A.V. Manohar, Effective field theories, in Schladming 1996, Perturbative and nonperturbative aspects of quantum field theory, p. 311-362, arXiv:hep-ph/9606222.

[9] See for example, O. Lauscher and M. Reuter, Ultraviolet fixed point and generalized flow equation of quantum gravity, Phys. Rev. D65 (2002) 025013 and arXiv:hep-th/0108040;

H. Gies, J. Jaeckel and C. Wetterich, Towards a renormalizable Standard Model without a fundamental Higgs scalar, Phys. Rev. D69 (2004) 105008 and arXiv:hep-ph/0312034.

[10] See for example P. Calabrese and A. Gambassi, Ageing properties of critical systems, J. Phys. A38 (2005) R133 and arXiv:cond-mat/0410357.

[11] See for example, D. Blas, D. Comelli, F. nesti and L. Pilo, Lorentz breaking massive gravity in curved space, arXiv;0905.1699 [hep-th].

[12] R.M. Hornreich, M. Luban and S. Shtrikman, Critical behavior at the onset of $\vec{k}$-space instability on the $\lambda$ line, Phys. Rev. Lett. 35 (1975) 1678;

See for example, M.M. Leite, Renormalization-group picture of the Lifshitz critical behavior, Phys. Rev. B 67 (2003) 104415.

[13] M.A. Shpot, Yu.M. Pis'mak and H.W.Diehl, Large- $n$ expansion for $m$-axial Lifshitz points, J. Phys. Condens. Matter 17 (2005) S1947 and arXiv:cond-mat/0412405.

[14] T.D. Bakeyev and A.A. Slavnov, Higher covariant derivative regularization revisited, Mod. Phys. Lett. A11 (1996) 1539 and arXiv:hep-th/9601092, and references therein.

[15] K.S. Stelle, Renormalization of higher derivative quantum gravity, Phys. Rev. D 16 (1977) 953;

E.S. Fradkin and A.A. Tseytlin, Renormalizable asymptotically free quantum theory of gravity, Nucl. Phys. B 201 (1982) 469.

[16] See for example, E.T. Tomboulis, Superrenormalizable gauge and gravitational theories, arXiv:hep-th/9702146.

[17] D. Anselmi and M. Halat, Renormalization of Lorentz violating theories, Phys. Rev. D 76 (2007) 125011 and arXiv:0707.2480 [hep-th].

[18] D. Anselmi, Weighted scale invariant quantum field theories, JHEP 02 (2008) 051 and arXiv:0801.1216 [hep-th].

[19] H.B. Nielsen and M. Ninomiya, Beta function in a noncovariant Yang-Mills theory, Nucl.Phys. B 141 (1978) 153;

S. Chadha and H.B. Nielsen, Lorentz invariance as a low-energy phenomenon, Nucl. Phys. B 217 (1983) 125.

[20] D. Anselmi, Weighted power counting, neutrino masses and Lorentz violating extensions of the Standard Model, Phys. Rev. D 79 (2009) 025017 and arXiv:0808.3475 [hep-ph].

[21] For reviews on the current status about neutrino masses, see A. Strumia and F. Vissani, Neutrino masses and mixings and..., arXiv:hep-ph/0606054;

M.C. Gonzalez-Garcia and M. Maltoni, Phenomenology of massive neutrinos, arXiv:0704.1800 [hep-ph]. 
[22] Y. Nambu and G. Jona-Lasinio, Dynamical model of elementary particles based on an analogy with superconductivity. I, Phys. Rev. 122 (1961) 345.

[23] D. Anselmi, Standard Model without elementary scalars and high energy Lorentz violation, arXiv:0904.1849 [hep-ph].

[24] O. Gagnon and G.D. Moore, Limits on Lorentz violation from the highest energy cosmic rays, Phys. Rev. D 70 (2004) 065002 and arXiv:hep-ph/0404196.

[25] P. Horava, Quantum gravity at a Lifshitz point, Phys. Rev. D 79 (2009) 084008 and arXiv:0901.3775 [hep-th].

[26] M. Li and Y Pang, A trouble with Horava-Lifshitz gravity, arXiv.0905.2751 [hep-th].

[27] V.A. Kostelecky, R. Potting, Gravity from spontaneous Lorentz violation, arXiv:0901.0662 [gr-qc].

[28] D. Anselmi, Weighted power counting and Lorentz violating gauge theories. I. General properties, Ann. Phys. 324 (2009) 874 and arXiv:0808.3470 [hep-th].

[29] D. Anselmi, Weighted power counting and Lorentz violating gauge theories. II. Classification, Ann. Phys. 324 (2009) 1058 and arXiv:0808.3474 [hep-th].

[30] V.A. Miransky, M. Tanabashi and K. Yamawaki, Is the $t$ quark responsible for the mass of $W$ and $Z$ bosons?, Mod. Phys. Lett. A4 (1989) 1043;

[31] W.A. Bardeen, C.T. Hill and M. Lindner, Minimal dynamical symmetry breaking of the standard model, Phys. Rev. D 41 (1990) 1647.

[32] A. Hasenfratz, P. Hasenfratz, K. Jansen, J. Kuti and Y. Shen, The equivalence of the top quark condensate and the elementary Higgs field, Nucl. Phys. B365 (1991) 79.

[33] Y. Nambu, in New Frontiers of Physics, proceedings of the XI International Symposium on Elementary Particle Physics, Kazimierz, Poland, 1988, Z. Ajduk, S. Pokorski and A. Trautman, Ed.s, World Scientific, Singapore, 1989.

[34] J. Goldstone, Field theories with superconductor solutions, Nuovo Cim. 19 (1961) 154;

J. Goldstone, S. Weinberg and A. Salam, Broken symmetries, Phys. Rev. 127 (1962) 965.

[35] M. Lindner, Top condensates as Higgs substitute, Int. J. Mod. Phys. A8 (1993) 2167.

[36] E. Farhi and L. Susskind, Technicolour, Phys. Rept. 74 (1981) 277; for more recent reviews, see C.T. Hill and E.H. Simmons, Strong dynamics and electroweak symmetry breaking, Phys. Rept. 381 (2003) 235 [Erratum-ibid. 390 (2004) 553];

and F. Sannino, Dynamical stabilization of the Fermi scale: phase diagram of strongly coupled theories for (minimal) walking technicolor and unparticles, arXiv:0804.0182 [hep-th].

[37] N. Maekawa, Electroweak symmetry breaking by vector-like fermions' condensation with small S and T parameters, Phys. Rev. D52 (1995) 1684;

G. Cynolter, E. Lendvai and G. Pócsik, Fermion condensate model of electroweak interactions, Eur. Phys. J. C46 (2006) 545 and hep-ph/0509230;

G. Cynolter, E. Lendvai and G. Pócsik, S and T parameters in the fermion condensate model, arXiv:0904.1090 [hep-ph]. 